\title{
Reflux after intravitreal injection: an anterior segment optical coherence tomography study
}

\begin{abstract}
Purpose: To investigate the intraocular pressure and conjunctival thickness changes following the intravitreal injection

Methods: Sixty eyes of 60 patients having intravitreal injection for age-related macular degeneration, macular edema associated with diabetes, central retinal vein occlusion, and branch retinal vein occlusion were enrolled. Intraocular pressure (IOP) was measured by Tonopen-Avia (Reichert Inc., NY, USA) in sitting position and five superior-temporal conjunctival images were obtained using the Anterior Segment 5 Line Raster scanning protocol of Cirrus HD-OCT 4000 (Carl Zeiss Meditec, Dublin, CA, USA) just before the intravitreal injection. $0.05 \mathrm{ml}$ bevacizumab with 27 -gauge needle, $0.05 \mathrm{ml}$ ranibizumab with 30-gauge needle, or dexamethasone implant with 23 -gauge needle was injected into the vitreous cavity. The second IOP measurements and OCT measurements were taken within 5 mins of injection.
\end{abstract}

Results: The ranibizumab group included 25 subjects, the bevacizumab group included 23 subjects, and the dexamethasone group included 12 subjects. IOP increases following intravitreal injection were significantly higher in ranibizumab and bevacizumab groups compared with Dexamethasone implant group $(\mathrm{p}<0.001$ and $\mathrm{p}=0.007$, respectively). Although, the increase of conjunctival thickness following the intravitreal injection was highest in Dexamethasone implant group, the differences between the groups did not reach statistically significance $(\mathrm{p}=0.153)$.

Conclusion: A higher IOP elevation is observed if a small-gauge needle is used for intravitreal injection. The conjunctival thickness changes following the intravitreal injection did not differ between the groups.

Keywords: intravitreal injection, conjunctival thickness, retinal vein occlusion, IOP elevation
Volume 10 Issue I - 2020

Ismail Ersan, Aydin Yildiz

Associate Professor, Department of Ophthalmology, Canakkale Onsekiz Mart University School of Medicine, Turkey

Correspondence: Ismail Ersan, Associate Professor, Department of Ophthalmology, Canakkale Onsekiz Mart University School of Medicine, Turkey, Tel 90505 8566574, Fax 902862180393 , Email isersa@gmail.com

Received: January II, 2020 | Published: February 07, 2020

\section{Introduction}

The intravitreal injection of various drugs is accepted as a safe intervention. Thus, use of antivascular endothelial growth factor (antiVEGF) and dexamethasone agents are used to treat several diseases of the posterior segment that are characterized by neovascularization or macular edema. ${ }^{1,2}$ Optical coherence tomography (OCT) is a noninvasive imaging technique that widely accepted by clinicians to evaluate the retina and choroid. ${ }^{3}$ Furthermore, various clinical applications of such as tear meniscus measurement, ocular surface disease (e.g., pterygium, pinguecula), Descemet's membrane detachment, corneal deposits (corneal dystrophies), keratitis, and glaucoma evaluation (angle assessment, morphological analysis of the filtering bleb after trabeculectomy). ${ }^{4}$ To the best of our knowledge, no study has examined the amount of vitreous reflux using anterior segment OCT. The purpose of present study was to evaluate the conjounctival thickness changes related with the amount of vitreous reflux.

\section{Methods}

This prospective observational study was approved by the university Ethics Committee and was compliant with the tenets of Declaration of Helsinki. This work was supported by Research Fund of the Canakkale Onsekiz Mart University. (TSA-2015-568) Informed consent was obtained before the participation. Sixty eyes of sixty patient having intravitreal injection for aged related macular degeneration (AMD), central retinal vein occlusion (CRVO), and branch retinal vein occlusion (BRVO) were enrolled to the study. Patients with pre-existing glaucoma, spherical equivalent greater than 1.00 diopters were excluded. Eligible patients were assigned to one of the injection group regarding to type of drugs intravitreally administrated. All participants underwent ophthalmic examinations, including best-corrected visual acuity, refractive error, slit-lamp evaluation, and fundus examination. Intraocular pressure (IOP) was measured by Tonopen-Avia (Reichert Inc., NY, USA) in sitting position and five superior-temporal conjunctival images were obtained using the Anterior Segment 5 Line Raster scanning protocol of Cirrus HD-OCT 4000 (Carl Zeiss Meditec, Dublin, CA, USA) just before the intravitreal injection. All injections were performed under sterile condition by the same surgeon. Under topical anesthesia with $\% 0.5$ proparakain (Alcaine ${ }^{\circledR}$, Alcon), periocular skins sterilized with $10 \%$ povidone-iodine; after the eye was draped with plastic adhesive drape and a lid speculum was inserted, $5 \%$ povidone-iodine were applied. $0.05 \mathrm{ml}$ bevacizumab (Avastin; Genentech, San Francisco, CA) with 27-gauge needle, $0.05 \mathrm{ml}$ ranibizumab (Lucentis; Novartis, Basel, Switzerland) with 30-gauge needle, or dexamethasone implant (Ozurdex, Allergan, Irvine, CA, USA) with 23-gauge needle was injected through the supero-temporal pars plana, 3.0-3.5 mm posterior of limbus into the vitreous cavity. Then, ophthalmic solution of moxifloxacin was administered. IOP measurements and OCT 
measurements were taken within 5 mins of injection, respectively. Patients with vitreous reflux requiring any tamponade were excluded. Statistical analysis was performed using the SPSS version 20.0. Simple comparisons between groups were performed using the nonparametric Mann-Whitney U test, and comparisons between several groups were performed using the Kruskal-Wallis test.

\section{Results}

The ranibizumab group included 25 subjects (14 males) with a mean age of $63.24 \pm 10.50$ (range, 43 to 82 years); the bevacizumab group included 23 subjects ( 12 males) with a mean age of $63.83 \pm 9.82$ (range, 40 to 83 years); the dexamethasone group included 12 subjects (5 males) with a mean age of $63.75 \pm 12.04$ (range, 37 to 79 years). The differences in age, gender, and lens status among the three groups were not statistically ( $\mathrm{p}=0.838,0.719$, and 0.191 , respectively) (Table 1). Diabetic macular edema was the most common reason for intravitreal injection in all the three groups (Table 1). IOP and conjunctival thickness measurements are shown in Table 2. Immediate postinjection IOP was compared among the groups, and it was found that Ranibizumab group had significantly higher postinjection IOP compared with Dexamethasone group $(\mathrm{p}<0.001)$. IOP increases following intravitreal injection were significantly higher in ranibizumab and bevacizumab groups compared with Dexamethasone implant group $(\mathrm{p}<0.001$ and $\mathrm{p}=0.007$, respectively). The serious complications such as endophthalmitis, retinal detachment, and vitreous hemorrhage, were not observed in any group. The numbers of patients with postinjection IOP values higher than $25 \mathrm{mmHg}$ in Ranibizumab and Bevacizumab groups were eight $(3.6 \%)$ and five $(2.2 \%)$, respectively. These patients were treated using $500 \mathrm{mg}$ of systemic oral acetazolamide. There was no patient with postinjection IOP value higher than $25 \mathrm{mmHg}$ in Dexamethasone implant group. Conjunctival thickness changes did not reach the statistically significance between the groups $(\mathrm{p}=0.169)$.

Table I Demographics and ocular characteristics of patients

\begin{tabular}{lllll}
\hline & Ranibizumab group & Bevacizumab group & Dexamethasone group & p \\
\hline Age & $63.24 \pm 10.50$ & $63.83 \pm 9.82$ & $63.75 \pm 12.04$ & 0.838 \\
Gender (male/female) & I4-Nov & I2-Nov & $05-\mathrm{Jul}$ & 0.719 \\
Lens status (phakic/pseudophakic) & Dec-13 & $17-\mathrm{Jun}$ & $07-$ May & 0.191 \\
Diagnosis & & & \\
Diabetic macular edema & $18(72.0 \%)$ & $16(69.6 \%)$ & $8(66.7 \%)$ \\
Age-related macular degeneration & $5(20.0 \%)$ & $2(8.7 \%)$ & $3(25.0 \%)$ \\
Central retinal vein occlusion & $2(8 \%)$ & $4(17.4 \%)$ & $1(8.3 \%)$ \\
Branch retina vein occlusion & - & $1(4.3 \%)$ & - \\
\hline
\end{tabular}

Table 2 Intraocular pressure and conjunctival thickness measurements

\begin{tabular}{|c|c|c|c|c|}
\hline & Ranibizumab group & Bevacizumab group & $\begin{array}{l}\text { Dexamethasone } \\
\text { group }\end{array}$ & $\mathbf{p}$ \\
\hline Preinjection IOP, $\mathrm{mmHg}$ & $\begin{array}{l}16.20 \pm 3,20 \\
(9,00-24,00)\end{array}$ & $\begin{array}{l}15,96 \pm 4,52 \\
(8,00-23,00)\end{array}$ & $\begin{array}{l}15,58 \pm 3,26 \\
(10,00-23,00)\end{array}$ & 0.538 \\
\hline Post injection IOP, $\mathrm{mmHg}$ & $\begin{array}{l}27,00 \pm 9,05^{*} \\
(15,00-49,00)\end{array}$ & $\begin{array}{l}22,70 \pm 9,23 \\
(8,00-49,00)\end{array}$ & $\begin{array}{l}16,08 \pm 4,10^{*} \\
(8,00-23,00)\end{array}$ & $<0.001$ \\
\hline IOP changes, $\mathrm{mmHg}$ & $\begin{array}{l}10.80 \pm 7,52^{*} \\
(2,00-30,00)\end{array}$ & $\begin{array}{l}7,30 \pm 8,22 ¥ \\
(-2,00-28,00)\end{array}$ & $\begin{array}{l}0,50 \pm 2,75^{*} \neq \\
(-4.00 \text { to } 4,00)\end{array}$ & $<0.001$ \\
\hline Preinjection conjunctival thickness, $\mu \mathrm{m}$ & $\begin{array}{l}249.04 \pm 65,51 \\
(103,00-430,33)\end{array}$ & $\begin{array}{l}258,03 \pm 46,30 \\
(193,33-384,67)\end{array}$ & $\begin{array}{l}259,39 \pm 52,73 \\
(183,33-325,67)\end{array}$ & 0.903 \\
\hline Post injection conjunctival thickness, $\mu \mathrm{m}$ & $\begin{array}{l}345.63 \pm 91,17 \\
(171,67-526,33)\end{array}$ & $\begin{array}{l}381,09 \pm 96,63 \\
(221,00-591,67)\end{array}$ & $\begin{array}{l}408.36 \pm 93.56 \\
(288,67-543.33)\end{array}$ & 0.169 \\
\hline Conjunctival thickness changes, $\mu \mathrm{m}$ & $\begin{array}{l}96.59 \pm 75,48 \\
(-2,33-218,33)\end{array}$ & $\begin{array}{l}123,06 \pm 78,73 \\
(-11.67-274.00)\end{array}$ & $\begin{array}{l}148,97 \pm 67,80 \\
(54.34-257.33)\end{array}$ & 0.153 \\
\hline
\end{tabular}

$*_{p}<0.001$ Bonferroni corrected Mann-Whitney $U$ test

$¥ p=0.007$ Bonferroni corrected Mann-Whitney $U$ test 


\section{Discussion}

Intravitreal drug injection is an effective route for retinal diseases. Also it allows the control of drug levels in the eye within a specified time. Physicians have a tendency to choose smaller gauge needles to decrease pain and injection related complications. The effect of injection technique related factors 55-10, axial length 10 , lens status6 on immediate IOP rise had extensively studied. Vitreous reflux was reported that the most important factor influencing the immediate postinjection IOP elevation. ${ }^{5}$ Thus, injection techniques, ${ }^{6}$ different needle sizes $^{7-15}$ were evaluated to decrease the vitreous reflux. Transient IOP increases following the anti-VEGF therapy have been studied extensively, whereas much is not known about the IOP increases following the DEX implantation. Previous studies concluded that the usage of smaller bore needles ${ }^{5}$ and the lack of vitreous reflux ${ }^{5}$ are related to higher immediate IOP spikes following the intravitreal injections. Pang et al. ${ }^{5}$ reported immediate IOP increase was found to be significantly lower in eyes injected with 30 -gauge needles compared with the smaller 32-gauge needles. Alagöz et al ${ }^{16}$ showed none of the eyes showed an immediate IOP increase of $\geq 5 \mathrm{mmHg}$ in patients having intravitreal injection of dexamethasone implant. In accordance with the literature ${ }^{17,18}$ we found that immediate IOP increases following intravitreal injection were significantly higher in ranibizumab using 30-gauge needle and bevacizumab groups using 27-gauge needle compared with Dexamethasone implant group $(\mathrm{p}<0.001$ and $\mathrm{p}=0.007$, respectively). We believe that higher IOPs in groups using smaller gauge needles were contributed by fewer vitreous reflux in smaller gauge needles. ${ }^{19}$

Various degrees of postinjection reflux is occasionally seen due to the intravitreal drug injection ${ }^{11}$ It was demonstrated that the reflux contains not only vitreous but also injected intravitreal drug 1912 The reflux of injected drug raises the concerns of loss of therapeutic dose. To address these concerns Brodie et al..$^{20}$ studied on cadaveric human eyes and reported the average loss of the original $50-\mu \mathrm{L}$ injection was only $0.74 \%$, with a maximum loss of $4.30 \%$. Using digital image analysis system in porcine eyes, the reflux contained some of the injected material but was predominantly composed of vitreous ${ }^{21}$ There was a higher incidence of postinjection reflux in eyes injected with 30 -gauge $(53 \%)$ compared with those injected with 32 -gauge (13\%, $\mathrm{P}=0.0007)$

Reflux was evaluated with the observation of presence or absence of conjunctival bleb formation, digital photographic technique, ${ }^{20}$ colorimetric comparison in enucleated bovine eyes,,$^{8,22,23}$ positron emission and computed tomography, ${ }^{24}$ cotton swab tes $\mathrm{t}^{25}$ measuring the conjunctival bleb diameter To the best of our knowledge, no study using anterior segment OCT has examined the subconjunctival blep due to the postinjection reflux. In accordance with the studies that reported a smaller needle bore size results in lower occurrence of vitreous reflux, we found a tendency of increasing conjunctival thickness changes with increasing the needle size, but the difference between the groups did not reach the clinically significance $(p=0.153) .{ }^{26-28}$ The limitations of this study is the small sample size, and the various variables that could not be controlled including status of vitreous, and the number of previous injections. One more limitation of the study is that only the conjunctival thickness measurements are used to indicate the amount of VR. Based on these results, we advise to take into account the immediate IOP increase in patient with a small amount of vitreous reflux. Future studies concerning advantages and disadvantages of using smaller gauge needles for intravitreal injection should be carried out as the physicians have tendency to favor smaller gauge needles.

\section{Acknowledgments}

This prospective study was supported by Research Fund of the Canakkale Onsekiz Mart University. (TSA-2015-568).

\section{Conflicts of interest}

Author declares that there is no conflict of interest.

\section{References}

1. Maggio E, Polito A, Guerriero M, et al. Intravitreal Dexamethasone Implant for Macular Edema Secondary to Retinal Vein Occlusion: 12-month Follow-Up and Prognostic Factors. Ophthalmologica. 2014;232(4):207-215.

2. Funk M, Karl D, Georgopoulos M, et al. Neovascular age-related macular degeneration: intraocular cytokines and growth factors and the influence of therapy with ranibizumab. Ophthalmology. 2009;116(12):2393-2399.

3. Mrejen S, Spaide RF. Optical coherence tomography: imaging of the choroid and beyond. Surv Ophthalmol. 2013;58(5):387-429.

4. Lim SH. Clinical Applications of Anterior Segment Optical Coherence Tomography. J Ophthalmol. 2015;2015:1-12.

5. Pang CE, Sorenson JA. Association between needle size, postinjection reflux, and intraocular pressure spikes after intravitreal injections. Retina. 2015;35(7):1401-1406.

6. Özkaya A, Alkın Z, Çelik U, et al. Comparing the Effects of Three Different Intravitreal Injection Techniques on Vitreous Reflux and Intraocular Pressure. J Ocul Pharmacol Ther. 2013;29(3):325-329.

7. Rodrigues EB, Meyer CH, Grumann A, et al. Tunneled Scleral Incision to Prevent Vitreal Reflux After Intravitreal Injection. Am J Ophthalmol. 2007;143(6):1035-1037.

8. De Stefano VS, Abechain JJ, de Almeida LF, et al. Experimental investigation of needles, syringes and techniques for intravitreal injections. Clin Exp Ophthalmol. 2011;39(3):236-242.

9. Soheilian M, Karimi S, Montahae T, et al. Effects of intravitreal injection of bevacizumab with or without anterior chamber paracentesis on intraocular pressure and peripapillary retinal nerve fiber layer thickness: a prospective study. Graefe's Arch Clin Exp Ophthalmol. 2017;255(9):1705-1712.

10. Arikan G, Osman Saatci A, Hakan Oner F. Immediate intraocular pressure rise after intravitreal injection of ranibizumab and two doses of triamcinolone acetonide. Int J Ophthalmol. 2011;4(4):402-405.

11. Benz MS, Albini TA, Holz ER, et al. Short-term course of intraocular pressure after intravitreal injection of triamcinolone acetonide. Ophthalmology. 2006;113(7):1174-1178.

12. Hubschman JP, Coffee RE, Bourges JL, et al. Experimental model of intravitreal injection techniques. Retina. 2010;30(1):167-173.

13. Kim JE, Mantravadi AV, Hur EY, et al. Short-term Intraocular Pressure Changes Immediately After Intravitreal Injections of AntiVascular Endothelial Growth Factor Agents. Am J Ophthalmol. 2008;146(6):930-934.

14. Jiménez-Gómez B, González-Montpetit M, Fonollosa Calduch A, et al Effects of ozurdex on intraocular pressure. A real life clinical practice study. Arch Soc Esp Oftalmol. 2015;90(9):421-425.

15. Hoang Q V, Jung JJ, Mrejen S, et al. Influence of axial length and postinjection reflux on sustained intraocular pressure elevation as a result of intravitreal anti-vascular endothelial growth factor therapy. Retina. 2014;34(3):519-524.

16. Alagöz N, Alagöz C, Yılmaz I, et al. Immediate Intraocular Pressure Changes Following Intravitreal Dexamethasone Implant. $J$ Ocul Pharmacol Ther. 2016;32(1):44-49. 
17. Uyar E, Ulas F, Sahin S, et al. Major factors affecting intraocular pressure spike after intravitreal ranibizumab injection: Vitreous reflux and its amount. Eur J Ophthalmol. 2019;29(4):361-367.

18. Bevacizumab İ, Sonrası E, Göz A. Immediate Intraocular Pressure Changes and Subconjunctival Reflux After Intravitreal Bevacizumab Injection: Comparison Between 27-Gauge and 30-Gauge Needle. 2014:273-277.

19. Boon CJF, Crama N, Klevering BJ, et al. Reflux after intravitreal injection of bevacizumab. Ophthalmology. 2008;115(7):1270.

20. Brodie FL, Ruggiero J, Ghodasra DH, et al. Volume and composition of reflux after intravitreal injection. Retina. 2014;34(7):1473-1476.

21. Brodie FL, Ruggiero J, Ghodasra DH, et al. A novel method for the measurement of reflux from intravitreal injections: data from 20 porcine eyes. Curr Eye Res. 2015;39(7):752-757.

22. Lorenz K, Zwiener I, Mirshahi A. Subconjunctival reflux and need for paracentesis after intravitreal injection of $0.1 \mathrm{ml}$ bevacizumab: comparison between 27-gauge and 30-gauge needle. Graefes Arch Clin Exp Ophthalmol. 2010;248(11):1573-1577.
23. Höhn F, Mirshahi A. Impact of injection techniques on intraocular pressure (IOP) increase after intravitreal ranibizumab application. Graefes Arch Clin Exp Ophthalmol. 2010;248(10):1371-1375.

24. Christoforidis JB, Williams MM, Epitropoulos FM, et al. Subconjunctival bleb that forms at the injection site after intravitreal injection is drug, not vitreous. Clin Exp Ophthalmol. 2013;41(6):614-615.

25. Lee CY, You YS, Lee SH, et al. Tower microneedle minimizes vitreal reflux in intravitreal injection. Biomed Microdevices. 2013;15(5):841-848.

26. Rodrigues EB, Grumann A, Penha FM, et al. Effect of needle type and injection technique on pain level and vitreal reflux in intravitreal injection. J Ocul Pharmacol Ther. 2011;27(2):197-203.

27. Uyar E, Ulas F, Sahin S, et al. Major factors affecting intraocular pressure spike after intravitreal ranibizumab injection: Vitreous reflux and its amount. Eur J Ophthalmol. 2019;29(4):361-367.

28. Kim K-S, Jee D. Effect of the Honan intraocular pressure reducer on intraocular pressure increase following intravitreal injection using the tunneled scleral technique. Jpn J Ophthalmol. 2011;55(6):632-637. 